\title{
Thermal Stress and Thermoregulation During Feeding in Mosquitoes
}

\author{
Chloé Lahondère and Claudio R. Lazzari \\ Additional information is available at the end of the chapter \\ http://dx.doi.org/10.5772/56288
}

\section{Introduction}

Many arthropods have acquired the ability to use the blood of endothermic vertebrates as their main or even unique food. Among insects, haematophagy has evolved independently in different groups [1], which have converged to this way of life under strong selective pressures that modelled many morphological, physiological and behavioural traits.

Blood is a rich source of nutrients and, except for the possible presence of parasites, otherwise sterile. However, being haematophagous is a risky task, as the food circulates inside vessels hidden beneath the skin of mobile hosts, able to defend themselves from biting or even predate on blood-sucking species. Thus, in order to minimize the contact with the host, blood-sucking insects need to pierce the host-skin without being noticed and gather blood in relatively high amounts and as quick as possible. Large blood-meals produce a strong osmotic misbalance at its ingestion and toxic metabolites as by products of its digestion. In addition, the rapid ingestion of a fluid which temperature can exceed that of the insects by $20^{\circ} \mathrm{C}$ or more and account for many times the insect's own body weight also implies a rapid transfer of heat into the insect's body. Thus, the inner temperature of the insect could exceed the physiological limits of certain functions, causing deleterious effects [2]. Numerous studies report the impact of temperature on different behavioural [3] and physiological processes such as development [4-6], metabolism [7, 8], blood-feeding and reproduction [9] of mosquitoes and insects in general.

Thermal stress may not only affect the insect itself but also its symbiotic flora [10-12] and the parasites that it transmits with an important impact on vector infectivity [13-15]. Finally, heat constitutes a main cue to find a food source (i.e. a warm-blooded vertebrate). Consequently, a recently fed insect could be exposed to cannibalism if its body temperature is higher than that 
of the surrounding environment, facilitating the horizontal transmission of parasites between vectors [16-17].

Provided their ectothermic nature, as well as their ability to colonize all kind of habitats, insects must cope with highly variable temperatures. Therefore, many insect species have developed particular physiological and behavioural mechanisms and strategies to avoid the risk to be submitted to thermal stress $[18,19]$. To avoid the effect of environmental heat, insects can seek for fresher environments or adjust their water loss to increase evaporation. In the case of haematophagous insects such as mosquitoes, they must in addition confront the exposition to thermal stress at each feeding event.

The problem of heat transfer between hosts and blood-sucking insects during blood feeding remained largely overlooked until recently, when unexpected physiological mechanisms against thermal stress were unravelled in mosquitoes. We present in this chapter a brief account of these findings and the perspectives that they open in both, fundamental and applied research.

\section{Thermal stress and protective strategies in Anopheles}

The first evidences of thermal stress during feeding in haematophagous insects were obtained only recently [20]. The variation of the temperature of the body during the feeding process was measured in different species of blood-sucking insects, including two mosquitoes, Aedes aegypti and Anopheles gambiae using thermocouples. As soon as feeding begins, a steady increase of the body temperature occurs, reaching peak values of up to $+10^{\circ} \mathrm{C}$ a few minutes later. After feeding, the temperature decreases gradually to come back similar to the environmental one. Depending on the values of environmental temperature, which is the initial temperature of the insect, and that of the blood, the amplitude and dynamics of heating and cooling vary.

Physiological responses of insects to heat include molecular changes, as is a rapid increase in the level of heat shock proteins (Hsps), which have a role as molecular chaperones that preserve the function of enzymes and other critical proteins [20]. More than a dozen Hsps are synthetized after exposure to high temperature, being the Hsp70 the most widely recognised as associated to thermal and other stresses. As in many other organisms, mosquito Hsp70s have been shown to increase during environmental stress [21, 22].

Benoit and co-workers [20] showed that, correlated with feeding and the associated elevation of the body temperature, a synthesis of heat-shock proteins occurs in Aedes aegypti in the few hours following a blood meal, in particular of Hsp70. In this species, the Hsp70 synthesis peaks 1 hour after feeding, reaching maximal expression in the mosquito midgut, where the relative amount of Hsp70 increases about 7 times after feeding. Similar increases in Hsp70 were showed immediately after blood feeding in Culex pipiens and in Anopheles gambiae, as well as in the bed bug Cimex lectularius. Nevertheless this increase, measured as the relative increase of mRNA by Northern blot, is not identical in the three mosquito species. Whereas in Aedes aegypti and 
Culex pipiens the relative level increases between nine and ten times, in Anopheles gambiae only three times. This last result is particularly interesting, since it is probable that the last named species would be less submitted to thermal stress, as we will discuss in the following sections.

\section{Heterothermy during feeding in Anopheles}

To better understand to what extent mosquitoes are exposed to thermal stress during feeding, we recently conducted a real-time infrared thermographic analysis of the evolution of the body temperature of Anopheles stephensi during feeding on live hosts at different skin temperatures and using an artificial feeder [23].

Thermal imaging analysis has first revealed that during feeding, the different regions of the mosquito's body exhibited different temperatures. When Anopheles stephensi fed on mice or human volunteers, their head temperature remained close to that of the ingested blood while the abdomen temperature stayed closer to that of the ambient temperature (Figure 1). The thermal profile along a mosquito's body during feeding, notwithstanding the exact temperature of the host skin, can be summarized as in this: $T_{\text {head }}^{\circ}>T_{\text {thorax }}^{\circ}>T_{\text {abdomen }}^{\circ}$. The fact of maintaining different temperatures in different regions of the body by an animal is named "regional heterothermy" and it is common in vertebrates living in cold aquatic or terrestrial environments. When the body temperature changes with time, this condition is called "temporal heterothermy". A combination of both types of heterothemy is frequently found in insects that perform pre-heating of flight muscles before taking off. By means of simultaneous isometric contractions of antagonist muscles, insects like bumble-bees and moths heat their thorax up to reach the optimal temperature for muscular work [18].

In the case of Anopheles stephensi, an average difference of $3.3^{\circ} \mathrm{C}$ between $T_{\text {head }}^{\circ}$ and $T_{\text {abdomen }}^{\circ}$ was measured when the $T_{\text {host }}^{\circ}$ was $34^{\circ} \mathrm{C}$ and $2.2^{\circ} \mathrm{C}$ when $T_{\text {host }}$ was $28^{\circ} \mathrm{C}$. At the end of feeding, when mouthparts are retracted from the skin, the mosquito temperature returns rapidly to environmental temperature (ectothermy).

Infrared thermography revealed a quite different pattern of body temperature in Aedes aegypti. In this species, the abdominal temperature during feeding remains close to that of the host, rather than to that of the environment as in Anopheles stephensi [23]. On the other hand, when the two species fed on sugar solution, despite the muscular activity of the ingestion pump, no heterothermy occurs: the temperature of the whole body remained that of the environment. As a consequence males, which don't feed on blood, exhibit a typically ectothermic thermal profile even when resting on a warm host, demonstrating that heating is only due to blood ingestion and not to the proximity of the host [23].

\section{Prediuresis and drop-keeping}

During blood feeding, most haematophagous species excrete drops of fluid, a process referred in mosquitoes as "prediuresis". The physiological function of prediuresis has been related to 


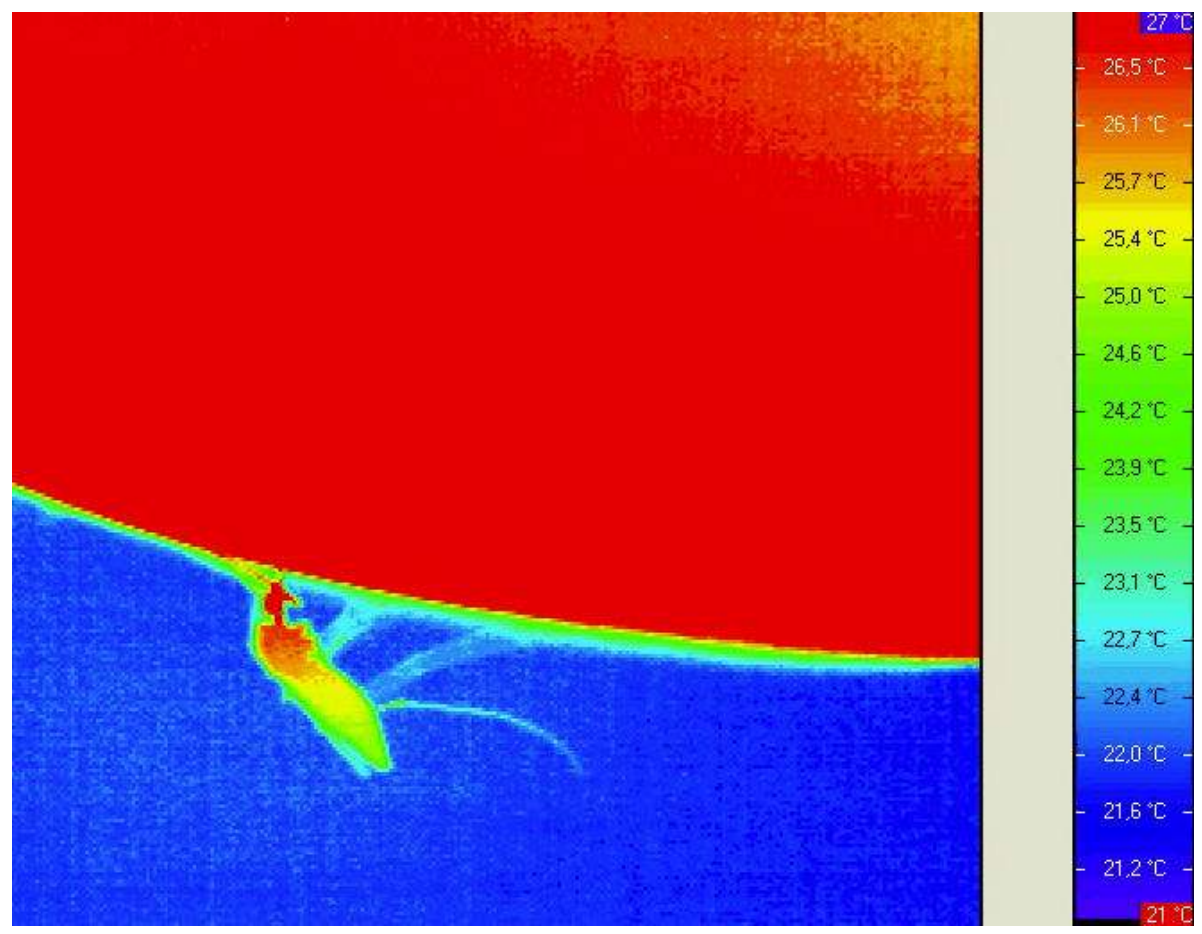

Figure 1. Thermographic image of an Anopheles stephensi female at the beginning of feeding on an anesthetized mouse $\left(T_{\text {host }}^{\circ}=28^{\circ} \mathrm{C}, T^{\circ}\right.$ environment $\left.=22^{\circ} \mathrm{C}\right)$. The temperature of the head is very close to the mouse one and a temperature gradient along the mosquito body can be observed (i.e., heterothermy).

erythrocytes concentration and elimination of water excess. The eliminated fluid is in most insects composed of urine, but in some blood-sucking species, such as mosquitoes and sandflies, it also contains fresh ingested blood that gives to the drop a bright red appearance. In mosquitoes, which feed not only on vertebrate blood, but also on nectar, prediuresis occurs during blood-feeding but it is rare or absent when they take a sugar meal.

In Anopheles stephensi, notwithstanding the nature of the host, blood-feeding almost always proceeds in a similar way: drops of fluid start being excreted during the first or second minute after the insect begins to feed. Frequently, a drop remains attached to the end of the abdomen for several minutes, increasing its size during feeding. Eventually the drop felt, and a new one is emitted and retained at the abdomen's end. The number of drops produced until complete gorging may vary.

Real-time thermography revealed that when Anopheles stephensi performs prediuresis and keeps a drop attached to its anus, a transient fall of $2^{\circ} \mathrm{C}$ or more of the abdominal temperature occurs and the characteristic heterothermy along its body becomes even more pronounced (Figure 2). The same phenomenon was observed in females of this species feeding in mice, human volunteers or using an artificial feeder [23]. Besides, when ingest- 
ing blood at the same temperature, the abdominal temperature of drop-keepers is significantly lower than that of mosquitoes that just perform prediuresis but that do not keep drops. These results demonstrate the existence of a physical cooling process in Anopheles stephensi. Conversely, drop-keeping was never observed to occur in Aedes aegypti among the individuals producing pre-urine while feeding, even if the frequency of prediuresis is the same in both mosquito species [23].

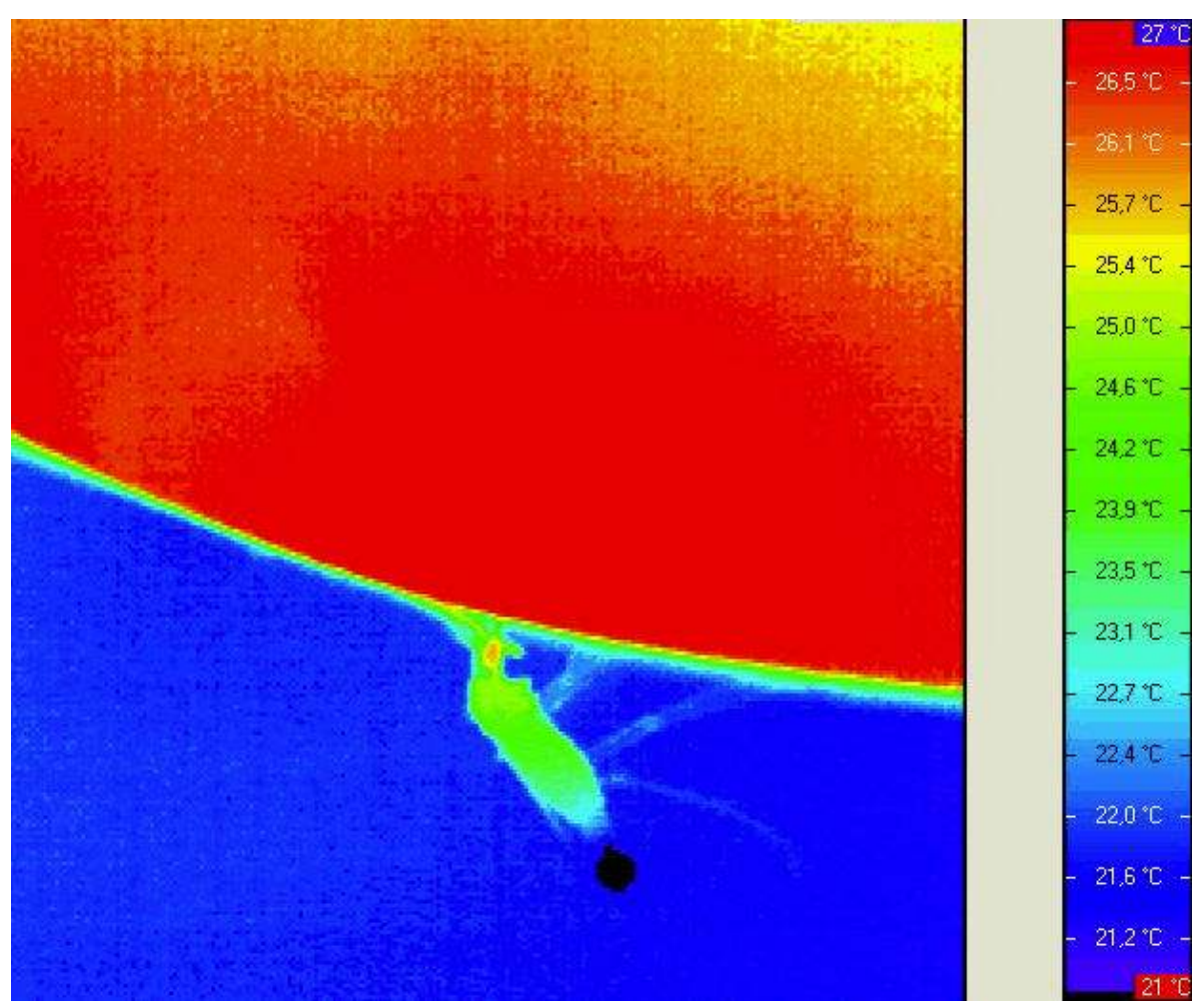

Figure 2. Thermographic image of the same Anopheles stephensi female as Figure 1, but during prediuresis. The mosquito performs evaporative cooling. The retention of the fluid drop attached to the abdomen end leads to a fall of the abdomen temperature causing a clear temperature gradient along the mosquito body. The colour of the droplet does not reflect the real temperature, because of the difference in the emissivity between the cuticle of the mosquito and the drop surface.

\section{Thermoregulation in Anopheles}

Many insects, in particular those having easy access to water, produce and retain drops of fluid, such as nectar, honey-dew, water or urine, depending on species, which evaporates in contact with the air, causing heat loss by evaporative cooling and the consequent decrease of 
the temperature of the insect body. Evaporative cooling constitutes an adaptive and effective response to risks associated to high temperature and has been observed in different groups of insects [24, 25].

This decrease of temperature helps them to avoid the deleterious physiological consequences of thermal stress. Some insects such as honeybees and bumblebees produce heat with their thoracic muscles while flying (endothermy) and regurgitate a droplet of nectar through their mouthparts to cool down their head, thus keeping the brain safe from overheating [26, 27]. Moths emit fluid, which is retained on the proboscis to refresh their head whereas others, like aphids, excrete honey-dew through their anus that consequently refresh their abdomen. The recorded loss of temperature is between 2 and $8^{\circ} \mathrm{C}$ depending on species [28].

\section{Anopheles stephensi feeding on a mouse at $35.5^{\circ} \mathrm{C}$}

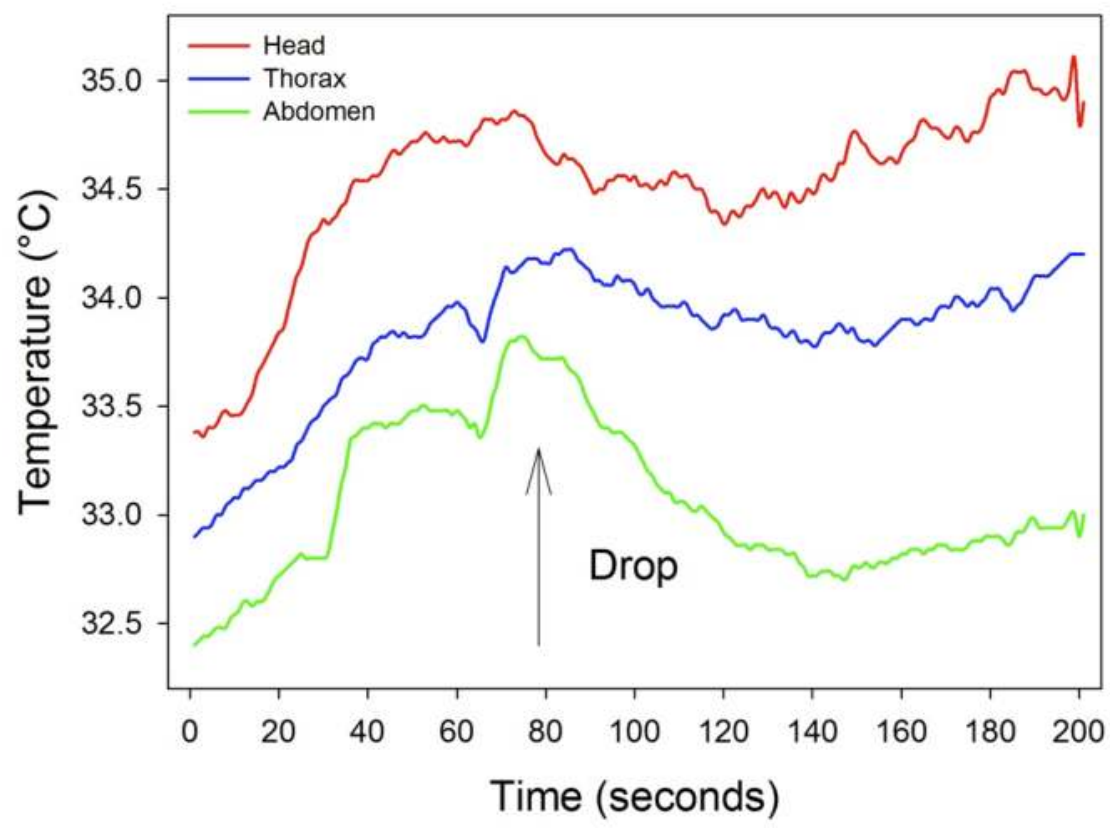

Figure 3. Evolution of the body temperatures of Anopheles stephensi during feeding on an anesthetized mouse. The arrow indicates the excretion of a droplet. $\left(T_{\text {host }}^{\circ}=35.5^{\circ} \mathrm{C}, T^{\circ}\right.$ environment $\left.=28^{\circ} \mathrm{C}\right)$

In Anopheles mosquitoes, the abdominal temperature of drop-keepers decreases of about $2^{\circ} \mathrm{C}$ during drop retention. For mosquitoes and in general for all haematophagous insects that need to manage an excess of water into their body during feeding and keep a well-adjusted water balance, evaporative cooling represents an efficient protective mechanism against overheating. 
To what extent prediuresis and drop-keeping occurs is variable and it is known that the rate of production and the size of the droplets excreted in mosquitoes during prediuresis differ not only between species but also within the same species, as also differs the amount of erythrocytes from the ingested blood [29].

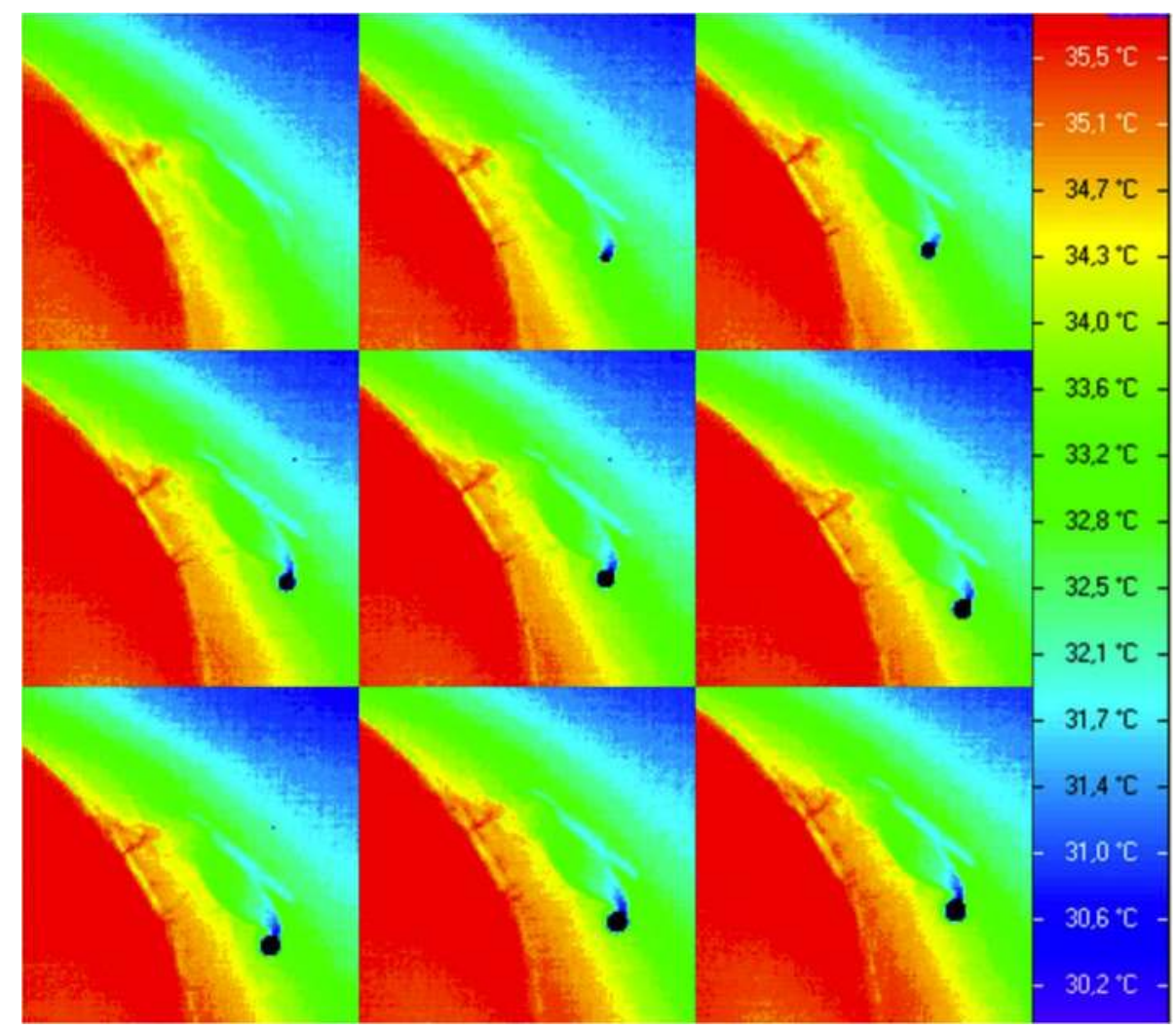

Figure 4. Sequence of thermographic images showing the production of a drop during feeding and the subsequent cooling of the abdomen in an Anopheles stephensi female. The insect fed on a human host $\left(T^{\circ}\right.$ host $=36^{\circ} \mathrm{C}, T^{\circ}$ environment $=$ $\left.23^{\circ} \mathrm{C}\right)$. Images were taken every 5 seconds.

\section{A novel significance of prediuresis}

Even though the occurrence of prediuresis and the elimination of fresh blood have been largely reported, it has been always considered just a way of concentrating erythrocytes and reducing the insect weight for take-off [30]. Nevertheless, two puzzling aspects of prediuresis in mosquitoes remained unsolved. The first one is the elimination during feeding of some of the just ingested blood containing erythrocytes [29]. It is widely accepted that strong selective 
pressures made blood-sucking insects minimize their contact time with a host in order to reduce the risk of being predated [1]. Thus, throwing away some of the food they ingest appears, at first glance, as a maladaptive strategy. From a point of view of thermoregulation, however, this "waste" makes sense, since it allows a quick increase in the volume (and evaporative surface) of the droplet and perhaps the surface properties of the drop, influencing its retention. Thus, the excretion of fresh blood during feeding in mosquitoes can be explained in terms of an adaptive response of evaporative cooling when exposed to thermal stress associated to feeding.

The second puzzling aspect of prediuresis is that not all mosquito species perform it. In fact, it has been shown that species that perform prediuresis need more time to reach repletion during a blood meal than species that do not produce pre-urine [31, 32]. Thus, the production of pre-urine could be seen, again, as a maladaptive strategy. However, an increase in feeding time could represent a trade-off between feeding quickly and avoiding overheating in species that are particularly sensitive to thermal stress. Others may be less sensitive or, as Aedes minimize the consequences of thermal stress by synthetizing more heat-shock proteins as, for example, Anopheles mosquitos.

Drop-keeping as evaporative cooling mechanism is in accordance with the particular position adopted by Anopheles species, which keep their abdomen away from the host surface. This causes the drop to be more exposed to the ambient air facilitating evaporation and cooling, and also avoiding the drop to be lost by contact with the host skin.

\section{Thermoregulation and pathogens transmission}

When anopheline mosquitoes ingest a blood meal from an infected host, mature and functional Plasmodium gametocytes are present in the erythrocytes and undergo differentiation in the mosquito midgut, a process that is influenced by temperature. Indeed, high temperatures negatively affect early stages of the parasite life cycle and no exflagellation occurs above $30^{\circ}$ C, holding parasites in an inactive state [14]. Later processes such as ookinete formation or migration of sporozoites towards the salivary glands are also influenced by temperature [15, 33, 34]. Furthermore, it has been well demonstrated that different species of Plasmodium are thermo-sensitive and that temperature has a direct impact on the incubation period of parasites in the mosquito [13]. On the other hand, the proliferation and dispersion of flaviviruses in Aedes mosquitoes is also under the influence of temperature but contrary to Plasmodium, this latter constitutes one of the most important factor positively influencing the extrinsic incubation period $(E I P)$. It has been shown that high temperatures are important for flaviviruses, acting on the rate of viral multiplication and consequently on the vector competence [35, 36, 37].

Moreover, Plasmodium parasites have to cope with the formation of the peritrophic matrix that follows each blood meal, which restrain their penetration through the gut wall [38, 39]. During the process of differentiation, Plasmodium ookinetes have to cross the peritrophic matrix and the midgut epithelium, before they turn into oocysts [40]. The time needed for the formation of the peritrophic matrix positively correlates with the vectorial capacity of mosquitoes, taking 
a longer time in Anopheles species than in species of Aedes or Culex [1, 41]. Thus, for Plasmodium parasites, insect's heterothermy could represent an important advantage, since when they enter into the mosquito's body, they are exposed to a rapid fall in temperature, which could immediately trigger exflagellation. Parasites could therefore penetrate the gut wall before the peritrophic matrix is fully formed.

From an evolutionary point of view, it makes sense that Plasmodium parasites take an advantage to be associated with species that undergo evaporative cooling, protecting them from lethal temperatures. On the other hand, flaviviruses associated with non drop-keeper species would benefit from a necessary warmer environment.

Evaporative cooling could also protect from heat stress the symbiotic microorganisms associated to mosquitoes and that can play an important role in haematophagous insects [10]. Asaia bacteria have been found in high density in the gut of Anopheles stephensi females as well as in ovaries [11]. Recently many genera have been identified in the midgut of natural populations of Anopheles gambiae [42]. In particular, the abundance of Enterobacteriaceae in the mosquito midgut has been found to correlate significantly with the Plasmodium infection status [42].

\section{Thermoregulation and thermotolerance in mosquitoes}

Finally, it is possible to speculate on two further implications of our interpretation of the functionality of prediuresis as thermoregulatory mechanisms. The first one concerns how environmental temperature may affect the survival of less thermotolerant mosquitoes. If we consider that the species that perform evaporative cooling could be more sensitive to heat, any change in the environmental temperature, due to local or global warming, would have a higher impact on them than on species that do not perform it, as for example Culex spp. that feed quickly and do not perform prediuresis while feeding [43]. It can be predicted that such species have been selected to reduce the contact time with their host and consequently to be more thermotolerant to temperature increases. Indeed, Aedes aegypti and its ability to produce Hsps represent an example of this [20].

The second implication of our finding is related to the control of mosquito populations. Prediuresis has deeper physiological consequences than just diuresis. In addition to excretion, it implies blood concentration and thermoregulation. The exploitation of the knowledge about excretion physiology to control disease vector insects by interfering with the function of Malpighian tubules has been already proposed for other haematophagous insects [44], and the same can be expected for mosquitoes. In this case, blocking or delaying the production of urine would have a double impact on disease transmission by affecting microorganisms transmitted by prediuresis $[45,46]$ and/or affecting the survival of mosquitoes exposed to overheating. 


\section{Conclusion}

Anopheles mosquitoes are capable to perform thermoregulation by evaporative cooling during blood intake. This mechanism protects the insect itself, as well as the associated microorganisms (both symbionts and parasites) from thermal stress. Thus, prediuresis which plays such different roles in the mosquito physiology, appears one more time as an interesting possible target for the control of disease vectors.

\section{Acknowledgements}

We are very grateful to Catherine Bourgouin and the CEPIA staff (Institut Pasteur, France) for providing us anopheline mosquitoes and rearing advices as well as Rogerio Amino (Institut Pasteur, France) for his valuable comments on the manuscript and helpful discussions. We also thank Fabrice Chandre and Marie-Noelle Lacroix (IRD Montpellier, France) for providing us Aedes aegypti eggs. This work received financial support from ANR (EcoEpi), CNRS and the University of Tours (France).

\section{Author details}

Chloé Lahondère and Claudio R. Lazzari*

*Address all correspondence to: claudio.lazzari@univ-tours.fr

Institut de Recherche sur la Biologie de l'Insecte, UMR CNRS - Université François Rabelais, Tours, France

\section{References}

[1] Lehane MJ. The biology of blood-sucking in insects Cambridge University Press, New York. $2^{\text {nd }}$ ed.; 2005.

[2] Kirby MJ, Lindsay SW. Responses of adult mosquitoes of two sibling species, Anopheles arabiensis and Anopheles gambiae s.s. (Diptera: Culicidae), to high temperatures. Bulletin of Entomological Research 2004; 94 441-448.

[3] Muirhead Thomson CR. The reactions of mosquitoes to temperature and humidity. Bulletin of Entomological Research 1938; 125-140. 
[4] Lanciani CA, Le TM. Effect of temperature on the wing length body-weight relationship in Anopheles quadrimaculatus. Journal of the American Mosquito Control Assocation 1995; 11 241-243.

[5] Lyimo EO, Takken W, Koella JC. Effect of rearing temperature and larval density on larval survival, age at pupation and adult size of Anopheles gambiae. Entomoligia Experimentalis et Applicata 1992; 63 265-271.

[6] Rueda LM, Patel KJ, Axtell RC, Stinner RE. Temperature-dependent development and survival rates of Culex quinquefasciatus and Aedes aegypti (Diptera: Culicidae). Journal of Medical Entomology 1980; 27 892-898.

[7] Clements AN. The biology of mosquitoes. Chapman \& Hall, London, UK, Vol. 1; 1992.

[8] Mellanby K. The influence of athmospheric humidity on the thermal death point of a number of insects. Journal of Experimental Biology 1932; 9 222-231.

[9] Eldridge BF. Effect of temperature and photoperiod on blood-feeding and ovarian development in mosquitoes of Culex pipiens complex. American Journal of Tropical Medicine and Hygiene 1968; 17 133-140.

[10] Brooks MA. Symbiotes and nutrition of medically important insects. Bulletin of the World Health Organisation 1964; 31 555-559.

[11] Favia G, Ricci I, Damiani C, Raddadi N, Crotti E, Marzorati M, Rizzi A, Urso R, Brusetti L, Borin S, et al. Bacteria of the genus Asaia stably associate with Anopheles stephensi, an Asian malarial mosquito vector. Proceeding of the National Academy of Science of the United States of America 2007; 104 9047-9051.

[12] Gusmão DS, Santos AV, Marini DC, Russo ES, Peixoto AMD, Bacci MJr, Berbert-Molina MA, Lemos, FJA. First isolation of microorganisms from the gut diverticulum of Aedes aegypti (Diptera: Culicidae): new perspectives for an insect-bacteria association. Memorias do Instituto Oswaldo Cruz 2007; 102 919-924.

[13] Boyd MF. Epidemiology: factors related to the definitive host. In Malariology. ed Boyd MF, W.B. Saunders \& Co, Philadelphia, Vol 1; 1949.

[14] Ogwan'g RA, Mwangi JK, Githure J, Were JBO, Roberts CR, Martin SK. Factors affecting exflagellation of in-vitro cultivated Plasmodium falciparum gametocytes. American Journal of Tropical Medicine and Hygiene 1993; 49 25-29.

[15] Vanderberg JP, Yoeli M. Effects of temperature on sporogonic development of Plasmodium berghei. Journal of Parasitology 1966; 52 559-564.

[16] Jones JC, Pilitt DR. Blood-feeding behavior of adult Aedes aegypti mosquitoes. Biological Bulletin 1973; 145 127-139.

[17] Weathersby AB, Ah HS, Mccall JW. Mosquitoes feeding on engorged mosquitoes. Mosquito News 1971; 31 110-111. 
[18] Heinrich, B. The hot-blooded insects : strategies and mechanisms of thermoregulation Harvard University Press, Cambridge, Massachusetts; 1993.

[19] May ML. Insect thermoregulation. Annual Review of Entomology 1979; 24 313-349.

[20] Benoit JB, Lopez-Martinez G, Patrick KR, Phillips ZP, Krause TB, Denlinger, DL. Drinking a hot blood meal elicits a protective heat shock response in mosquitoes. Proceeding of the National Academy of Science of the United States of America 2011; 108 8026-8029.

[21] Gross TL, Myles KM, Adelman ZN. Identification and characterization of heat shock 70 genes in Aedes aegypti (Diptera: Culicidae). Journal of Medical Entomology 2009; 46 496-504.

[22] Benoit JB, Lopez-Martinez G, Phillips ZP, Patrick KR, Denlinger DL. Heat shock proteins contribute to mosquito dehydration tolerance. Journal of Insect Physiology 2010; 56 151-156.

[23] Lahondère C, Lazzari CR. Mosquitoes cool down during blood feeding to avoid overheating. Current Biology 2012; 22, 40-45.

[24] Adams PA, Heath JE. An evaporative cooling mechanism in Pholus achemon. Journal of Research on the Lepidoptera 1964; 3 69-72.

[25] Mittler TE. The excretion of honey-dew by Tuberolachnus salignus (Gmelin) (Homoptera: Aphididae). Proceeding of the Royal Entomological Society, Series A General Entomology 1958; 33 49-55.

[26] Heinrich B. Heat-exchange in relation to blood-flow between thorax and abdomen in bumblebees. Journal of Experimental Biology 1976; 64 561-585.

[27] Heinrich B. Keeping a cool head - honeybee thermoregulation. Science 1979; 205 1269-1271.

[28] Prange HD. Evaporative cooling in insects. Journal of Insect Physiology 1996; 42 493-499.

[29] Chege GMM, Beier JC Blood acquisition and processing by three Anopheles (Diptera: Culicidae) species with different innate susceptibilities to Plasmodium falciparum. Journal of Medical Entomology 1998; 35(3) 319-323.

[30] Briegel H, Rezzonico L. Concentration of host blood protein during feeding by Anopheline mosquitoes (Diptera, Culicidae). Journal of Medical Entomology 1985; 22 612-618.

[31] Vaughan JA, Noden BH, Beier JC. Concentration of human erythrocytes by Anopheline mosquitoes (Diptera, Culicidae) during feeding. Journal of Medical Entomology 1991; 28 780-786. 
[32] Chadee DD, Beier JC, Mohammed RT. Fast and slow blood-feeding durations of Aedes aegypti mosquitoes in Trinidad. Journal of Vector Ecology 2002; 27 172-177.

[33] Ball GH, Chao J. Temperature stresses on mosquito phase of Plasmodium relictum. Journal of Parasitology 1964; 50 748-752.

[34] Noden BH, Kent MD, Beier JC. The impact of variations in temperature on early Plasmodium falciparum development in Anopheles stephensi. Parasitology 1995; 111 539-545.

[35] Davis NC. The effect of various temperatures in modifying the extrinsic incubation period of the yellow fever virus in Aedes aegypti. American Journal of Epidemiology 1932; 16 163-176.

[36] Hardy JL, Houk EJ, Kramer LD, Reeves WC. Intrinsic factors affecting vector competence of mosquitoes for arboviruses. Annual Review of Entomology 1983; 28 229-262.

[37] Kramer LD, Ebel GD. Dynamics of flavivirus infection in mosquitoes. Advances in Virus Research 2003; 60 187-232.

[38] Baton LA, Ranford-Cartwright LC. How do malaria ookinetes cross the mosquito midgut wall? Trends in Parasitology 2005; 21 22-28.

[39] Freyvogel TA, Staeubli W. The formation of the peritrophic membrane in Culicidae. Acta Tropica 1965; 22 118-147.

[40] Sinden RE. Plasmodium differentiation in the mosquito. Parassitologia 1999; 41 139-148.

[41] Devenport M, Jacobs-Lorena M. The peritrophic matrix of hematophagous insects. In Biology of Disease Vectors, ed. W.C. Marquardt Elsevier Academic Press, Amsterdam; 2005.

[42] Boissière A, Tchioffo MT, Bachar D, Abate L, Marie A, Nsango SE, Shahbazkia HR, Awono-Ambene PH, Levashina EA, Christen R, Morlais I. Midgut microbiota of the malaria mosquito vector Anopheles gambiae and interactions with Plasmodium falciparum infection. Public Library of Science Pathogens 2012; 8(5): e1002742. doi: 10.1371/ journal.ppat.1002742

[43] Vaughan JA, Azad AF Passage of host immunoglobulin-G from blood meal into hemolymph of selected mosquito species (Diptera, Culicidae). Journal of Medical Entomology 1988; 25 472-474.

[44] Santini MS, Ronderos JR. Allatotropin-like peptide released by Malpighian tubules induces hindgut activity associated with diuresis in the Chagas disease vector Triatoma infestans (Klug). Journal of Experimental Biology 2007; 210 1986-1991.

[45] Sadlova J, Reishig J, Volf P. Prediuresis in female Phlebotomus sandflies (Diptera: Psychodidae). European Journal of Entomology 1998; 95 643-647. 
[46] Blow JA, Turell MJ, Walker ED, Silverman AL. Post-bloodmeal diuretic shedding of hepatitis B virus by mosquitoes (Diptera: Culicidae). Journal of Medical Entomology 2002; 39 605-612. 